\title{
A comparative study of tree community structure and natural regeneration status in Bontang urban forest and conservation forest of the LNG Industrial Plant Area, East Kalimantan, Indonesia
}

\author{
SUDRAJAT SUDRAJAT ${ }^{1, \vartheta}$, MINTORO DWIPUTRO ${ }^{2, \bullet \bullet}$ \\ ${ }^{1}$ Laboratory of Ecology and Biodiversity, Faculty of Mathematics and Natural Sciences, Universita Mulawarman. Jl. Barong Tongkok Gn. Kelua, \\ Samarinda 75242, East Kalimantan, Indonesia. Tel.: +62-541-749140, `email: sudrajat.fmipa @ gmail.com. \\ ${ }^{2}$ Laboratory of Plant Anatomy and Systematics, Faculty of Mathematics and Natural Sciences, Universita Mulawarman. J1. Barong Tongkok Gn. Kelua, \\ Samarinda 75242, East Kalimantan, Indonesia. Tel.: +62-541-749140, •`email: mintorodwi@ gmail.com
}

Manuscript received: 5 August 2019. Revision accepted: 5 September 2019.

\begin{abstract}
Sudrajat S, Dwiputro M. 2019. A comparative study of tree community structure and natural regeneration status in Bontang urban forest and conservation forest of the LNG Industrial Plant Area, East Kalimantan, Indonesia. Biodiversitas 20: 2841-2847. The objective of this study was to describe and compare the community structure and natural regeneration status of tree species in urban forests within industrial estates. This study was conducted in two types of forests, Bontang urban forests within industrial area and conservation forests in the Industrial buffer zone area. At each forest location, a transect was made, and along the transect, a plot of size 20 x 20 meters was made with a distance between plots of 100 meters. In each plot, several subplots were made with size of $5 \mathrm{~m} \times 5 \mathrm{~m}$ for sapling level, and $2 \mathrm{~m} \times 2 \mathrm{~m}$ for seedling level. The results of this study showed that in urban forest areas there were 32 tree-level species, 21 sapling species and 15 seedling species, belonging of 49 genera and 39 families. The number of species, species richness, species diversity, evenness of sapling and tree-level species was more in urban forests than in conservation forest, and the condition was vice versa for seedling level. The value of species dominance index in conservation forest was greater than urban forest, and conversely, the value of individual density/ha for tree-level was 562.50 individuals/ha, sapling level was 7,933 ind./Ha and for seeding level 80,625 ind./ha. Of the 53 urban forest species, 28 species (52.83\%) were in no regeneration category, 3 species $(5.66 \%)$ were in poor regeneration category, and 22 species $(41.50 \%)$ were in the new species category. In contrast, among 76 species of the conservation forests, 8 species $(10.52 \%)$ showed good regeneration status, 13 species $(17.10 \%)$ showed poor status and 55 species $(72.36 \%)$ showed no regeneration status. From the results, it can be concluded that the Bontang urban forest in the industrial area shows no regeneration status and the emergence of new species. In the context of conservation, it is recommended to implement special silvicultural techniques to maintain the biodiversity of this urban forest.
\end{abstract}

Keywords: Industrial estate, natural regeneration, tropical rainforest, urban forest

\section{INTRODUCTION}

The Bontang urban forestry, which is located in the industrial area of a liquefied natural gas refinery, is considered as an ecosystem that has rich and unique flora and fauna, and many commercially and historically valuable tree species, as the rest of the lowland tropical forest ecosystems of East Kalimantan. This forest is the result of fragmentation since 1972 from its main habitat, Kutai National Park. The urban forest is currently very well-protected and keeps showing a picture of the lowland type of Dipterocarpaceae primary forest. This condition is due to the the very tight security provided by the company's security forces for this region.

Based on the estimated age of trees carried out by Zuhud et al. (1995), there exists several species of trees older than 100 years, such as Canarium caudatum., Litsea spp., Bischofia spp., Eugenia spp., Lophopetalum spp., Ficus spp. etc. The estimated age of a Canarium caudatum tree is 535 years. There are 18 protected species located in this area, namely: Alstonia scholaris, Aquilaria malaccensis, Diospyros buxifolia, Diospyros coriacea,
Diospyros rigidus, Diospyros sumatrana, Diospyros toposioides, Drybalanops sp., Duabanga moluccana, Dyera costulata, Eurycoma longifolia, Eusideroxylon zwageri, Fagraea fragrans, Flacourtia rukam, Ganua motleyana, Lophopetalum beccarianum, Shorea leprosula, and Styrax macrocarpa. From the inventory, theoretically, there are potential areas for other planting activities in this urban forest area.

Mishra et al. (2013) has stated that the assessment of the structure and composition of forest communities is very important to understand the condition of tree populations, their regeneration capabilities.. Natural regeneration is one of the factors that can change the stand structure of tree species growing, the number of trees, the location and composition of trees over time (Kusmana and Susanti 2015). Therefore, understanding the status of regeneration and how it influences the abiotic and biotic factors is important for ecological studies. The natural ability of forests to regenerate species is the key to the ecosystem to be able to continue carrying out its functions. The process of regenerating a species is largely determined by the seed production process and its spread, germination power, and 
its growth power from seed to adulthood. Regeneration is a recovery process that occurs in the forest where trees that have died naturally or due to wind exposure, flooding, logging will be replaced by new individuals. Dead trees that have seed banks, will be replaced by seedlings of the same plant species. Light tolerant species regenerate quickly and survive into adulthood. But the seedlings of light intolerant species will experience dormancy and regeneration will occur only under shaded conditions.

Maintenance of forest stands with sufficient regeneration is the main target in conservation ecology. The existence of strict protection by industrial companies for fragments of the urban forest is very helpful for ensuring their sustainability, but because of their isolation from original forest, reproductive capacity may be affected resulting in a typical regeneration status. Information about the structure and composition of the community as well as the regeneration status of this forest type has never been reported. It is also very interesting to study how the community constituents of isolated forest fragments and the regeneration ability of each species in them. The results of this study are expected to be helpful inputs for determining management strategies and policies of Bontang urban forest areas in order to ensure their sustainability.

\section{MATERIALS AND METHODS}

\section{Location and characteristics of study areas}

Geographically, Bontang City is located between $117^{\circ} 23^{\prime}$ and $117^{\circ} 38^{\prime}$ East and between $0^{\circ} 01^{\prime}$ and $0^{\circ} 12^{\prime}$ North. The location of Bontang Urban Forest is in the area of PT Badak Natural Gas Liquefaction covering \pm 7.4 hectares, and located in geographical coordinate points of $\mathrm{N} 0^{\circ} 06{ }^{\prime} 5^{\prime}$ and E $117^{\circ} 30^{\prime}$ (Figure 1). This area was designated as Bontang City forest on 31 May 2012 based on the Bontang Government Decree No. 259/2012 on Determination of Natural Forests of PT. Badak NGL as Green Open Space or Urban Forest.

The soil type is dominated by yellow, alluvial and latosol complex red pod solid. This soil has topsoil that is thin and sensitive to erosion and nutrient-poor. Based on the Schmidt and Ferguson classification, this region has type B climate with Q values ranging from 14.3 to $33.3 \%$. From the data at the observation station in the Bontang area, the average annual rainfall is $1543.6 \mathrm{~mm}$ and the average temperature is $26^{\circ} \mathrm{C}$ (ranging from 24 to $33^{\circ} \mathrm{C}$ ) with a relative humidity of $67-98 \%$.

\section{Sampling method}

Sampling is carried out in the Bontang urban forest (Location A) which is in the Liquefied Natural Gas plant environment and the conservation forest managed by the company in the buffer zone between the company area and the outside environment (Location B). Field data collection is carried out with a plot transect system to make a path along the field contour. The lines are made of 6 transects (2 transects at Location A and 4 transects at location B) with a length of $500 \mathrm{~m}$ or more and $20 \mathrm{~m}$ wide, with a distance of $200 \mathrm{~m}$ in between. In the transect, observation plots are made intermittently with a distance of $100 \mathrm{~m}$ between each plot. In each plot, several subplots are made, namely a $20 \mathrm{~m}$ x $20 \mathrm{~m}$ plot for tree-level samples, $5 \mathrm{~m}$ x $5 \mathrm{~m}$ for the sapling level, and $2 \mathrm{~m} \times 2 \mathrm{~m}$ for seedling levels.
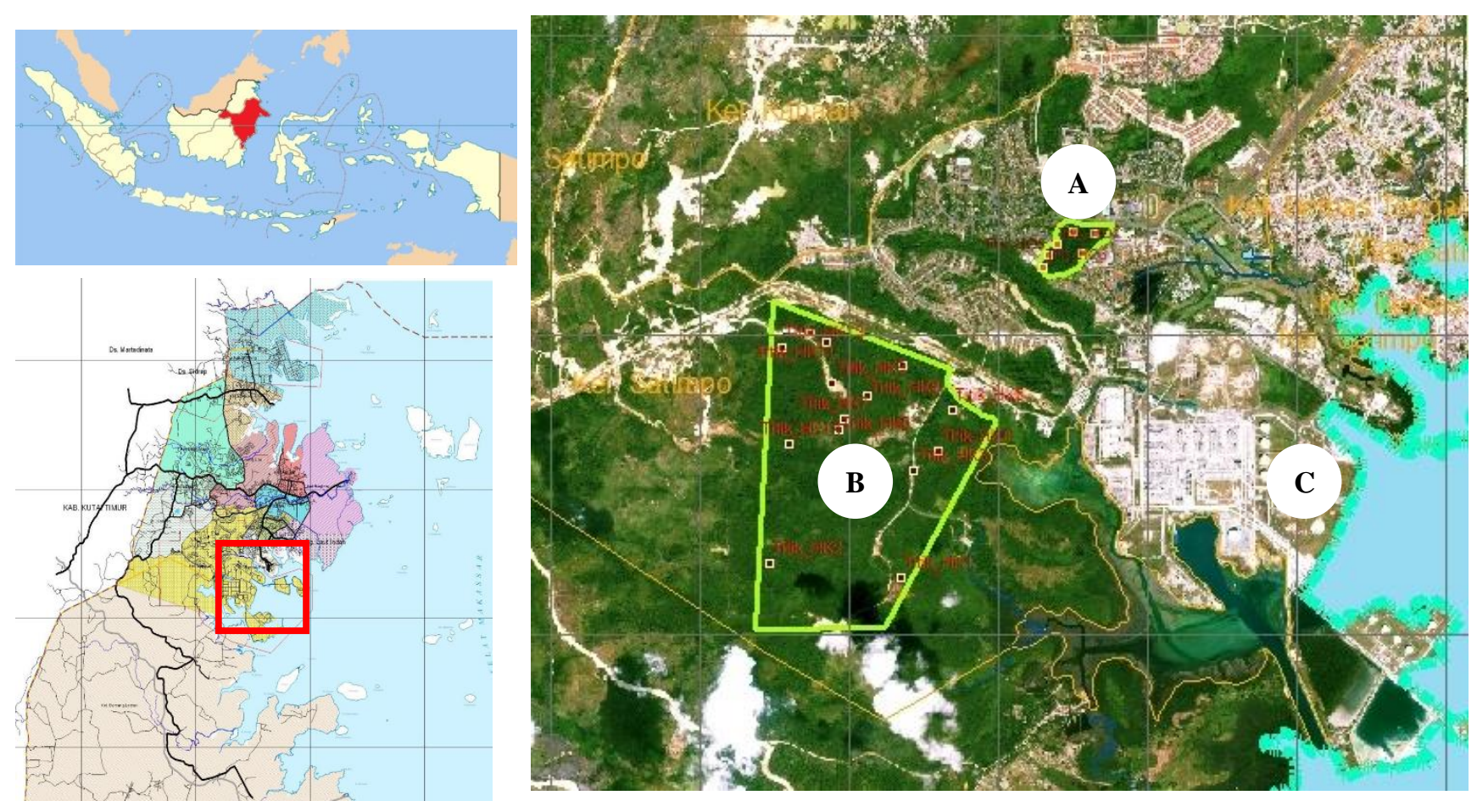

Figure 1. Study plots at Urban Forests in the liquefied natural gas industry area in Bontang, East Kalimantan, Indonesia. A. Natural forest isolated in the area of LNG plant, B. Company conservation forest area, C. Operational area ofl natural gas liquefaction plant 
Along the paths in the plot, the types found from the lower plants to the large and small trees are recorded. Plants are divided into seedlings, saplings and trees (Pudyatmoko et al. 1997; Sing et al. 1986; Dhar et al. 1997; Kusmana 1997; Ngo et al. 2017). The size class categories are "seedling level", i.e. the regeneration of tree species (dbh $<2 \mathrm{~cm}$ and height $<150 \mathrm{~cm}$ ); "Sapling level", i.e. above $150 \mathrm{~cm}$ high and chest height $>2 \mathrm{~cm}$ to $<10 \mathrm{~cm}$, and "tree level", i.e. diameter at breast height $>10 \mathrm{~cm}$. The species of plants that cannot be identified in the fields, are identified using herbarium, in the laboratory of the Dendrology Faculty of Forestry University Mulawarman Samarinda.

\section{Data analysis}

The value of density, frequency and important values are analyzed based on the formula of Cox (1976). The diversity of tree species is calculated based on (i) diversity index of Shannon (H'), and (ii) evenness index (MuellerDombois and Ellenberg 1974). Species importance level in each location is calculated by adding relative values of frequency, density and dominance. Data used for the analysis of tree species regeneration status consists of family, species name, number of trees, the population of sapling and seedling levels of each species. Criteria used for assessing species regeneration status was according to Malik and Bhatt (2016) which is as follows: tree species are declared as having "good" regeneration status, if seedling level population $>$ tree>, "fair" if seedling level population $>$ or $\leq$ stake $\leq$ tree, "poor" if there is only sapling level individuals, no seedlings (seedlings $<$,> or $=$ tree), "no regeneration" if there is only tree level, saplings and seedlings are not found, and "newcomer species" if there are no trees but only saplings and or seedlings .

\section{RESULTS AND DISCUSSION}

\section{Forest community composition and structure}

Analysis of species composition in the sampling plots of two remnant forest fragments studied (Locations A and $B)$, revealed that the number of tree species ranged between 10 and 32, number of sapling level species ranged between 3 and 21, and sapling level ranged between 7 and 34. The values of density, relative density, relative frequency, species richness, important values of species, species diversity index, dominance index, and evenness index are presented in Table 1. Based on the data in Table 1, forest fragments in buffer zones of industrial forest conservation companies had an average density of 491 trees/ha, 3,725 saplings/ha and 69,218 seedlings/ha, , and 562 trees/ha, 7,933 saplings/ha, and 80,625 seedlings/ha, in Bontang urban forest.

Table 1. Structure and composition of trees in the Bontang Urban Forest of the Industrial and Conservation Forest of the buffer zone of the Liquid Natural Gas Industry area, East Kalimantan, Indonesia

\begin{tabular}{|c|c|c|c|c|c|c|c|c|}
\hline \multirow{3}{*}{ Parameter } & \multicolumn{8}{|l|}{ Location } \\
\hline & \multicolumn{4}{|c|}{ Conservation Forest in Buffer Zone } & \multicolumn{4}{|c|}{ Bontang Urban Forest } \\
\hline & 1 & 2 & 3 & 4 & Average & 1 & 2 & Average \\
\hline \multicolumn{9}{|l|}{ Total Species } \\
\hline Seedling & 34 & 15 & 26 & 7 & 20.50 & 15 & 14 & 14.50 \\
\hline Sapling & 20 & 15 & 15 & 3 & 13.25 & 17 & 21 & 19.00 \\
\hline Tree & 20 & 32 & 39 & 10 & 25.25 & 32 & 27 & 29.50 \\
\hline \multicolumn{9}{|c|}{ Important species index values (\%) } \\
\hline Seedling & 200 & 200 & 200 & 200 & 200 & 200 & 200 & 200 \\
\hline Sapling & 200 & 200 & 200 & 200 & 200 & 200 & 200 & 200 \\
\hline Tree & 100 & 300 & 300 & 300 & 250 & 300 & 300 & 300 \\
\hline \multicolumn{9}{|c|}{ Density $($ Ind/Ha) } \\
\hline Seedling & 100,625 & 92.500 & 35,000 & 48,750 & 69,218 & 50.000 & 111.250 & 80,625 \\
\hline Sapling & 4,500 & 5,200 & 4,400 & 800 & 3,725 & 7,467 & 8,400 & $7,933.50$ \\
\hline Tree & 444 & 525 & 550 & 488 & 491,75 & 625 & 500 & 562.50 \\
\hline \multicolumn{9}{|c|}{ Species Richness Index $(R)$} \\
\hline Seedling & 6.49 & 2.97 & 6.21 & 1.64 & 4.32 & 3.42 & 2.90 & 3.16 \\
\hline Sapling & 4.99 & 3.82 & 3.70 & 1.44 & 3.48 & 3.97 & 5.35 & 4.66 \\
\hline Tree & 4.66 & 7.48 & 8.49 & 2.46 & 5.77 & 7.18 & 7.32 & 7.25 \\
\hline \multicolumn{9}{|c|}{ Species diversity index ( $\left.H^{\prime}\right)$} \\
\hline Seedling & 2.95 & 2.01 & $2, .8$ & 1.64 & 2.39 & 2.33 & 1.64 & 1.98 \\
\hline Sapling & 2.70 & 2.45 & 2.35 & 1.04 & 2.13 & 2.42 & 2.74 & 2.58 \\
\hline Tree & 2.38 & 2.99 & 3.31 & 2.01 & 2.67 & 3.12 & 3.23 & 3.17 \\
\hline \multicolumn{9}{|c|}{ Dominance Index C) } \\
\hline Seedling & 0.09 & 0.21 & 0.07 & 0.23 & 0.15 & 0.13 & 0.30 & 0.21 \\
\hline Sapling & 0.09 & 0.11 & 0.13 & 0.38 & 0.17 & 0.13 & 0.09 & 0.11 \\
\hline Tree & 0.15 & 0.08 & 0.05 & 0.16 & 0.11 & 0.06 & 0.04 & 0.05 \\
\hline \multicolumn{9}{|c|}{ Species evenness index (e) } \\
\hline Seedling & 0.84 & 0.74 & 0.92 & 0.84 & 0.83 & 0.86 & 0,62 & 0.74 \\
\hline Sapling & 0.90 & 0.90 & 0.87 & 0.95 & 0.90 & 0.85 & 0.90 & 0.87 \\
\hline Tree & 0.80 & 0.86 & 0.90 & 0.87 & 0.85 & 0.90 & 0.97 & 0.93 \\
\hline
\end{tabular}

Note: 1,2,3: Numbers of observation transects 
Table 2. Regeneration status of tree species of Bontang Urban Forest in the Industrial Area of the Liquid Natural Gas Industry, East Kalimantan, Indonesia

\begin{tabular}{|c|c|c|c|c|c|c|}
\hline \multirow[t]{2}{*}{ No } & \multirow[t]{2}{*}{ Family } & \multirow[t]{2}{*}{ Species } & \multicolumn{3}{|c|}{ Density (ind/ha) } & \multirow{2}{*}{$\begin{array}{l}\text { Status } \\
\text { regeneration }\end{array}$} \\
\hline & & & Tree & Sapling & Seedling & \\
\hline 1 & Lecythidaceae & Planchonia grandis Ridl & 75 & & & No \\
\hline 2 & Apocynaceae & Alstonia scholaris (L.) R. Br & 33 & & & No \\
\hline \multirow[t]{7}{*}{3} & Hypericaceae & $\begin{array}{l}\text { Cratoxylum formosum (Jack) Benth. \& Hook.f. } \\
\text { ex Dyer. }\end{array}$ & 83 & 267 & & poor \\
\hline & Dipterocarpaceae & Vatica umbonata (Hook.f.) Burck. & 25 & & & No \\
\hline & Dipterocarpaceae & Shorea smithiana Sym. & 17 & & & No \\
\hline & Dipterocarpaceae & Shorea leprosula Miq. & 17 & & & No \\
\hline & Dipterocarpaceae & Anisoptera costata Korth. & 8 & & & No \\
\hline & Dipterocarpaceae & Shorea ovalis Blume & 8 & & & No \\
\hline & Dipterocarpaceae & Shorea elliptica Burck. & & & 833 & New \\
\hline 5 & Theaceae & Schima wallichii (DC.) Korth & 33 & & & No \\
\hline 6 & Chrysobalanaceae & Licania splendens (Korth.) Prance \& Kosterm & 25 & & & No \\
\hline \multirow[t]{2}{*}{7} & Rubiaceae & Breonia chinensis (Lam.) Capuron & 17 & & & No \\
\hline & Rubiaceae & Tarenna costata Merr. & 8 & & & No \\
\hline \multirow[t]{2}{*}{8} & Lauraceae & Actinodaphne macrophylla (Blume) Nees. & 17 & & & No \\
\hline & Lauraceae & Litsea elliptica Blume & & 400 & & New \\
\hline 9 & Centroplacaceae & Bhesa paniculata Arn. & 17 & & & No \\
\hline 10 & Burceraceae & Dacryodes rostrata (B1.) H.J. Lam & 17 & & & No \\
\hline 11 & Clusiaceae & Garcinia beccarii Pierre. & 8 & & & No \\
\hline 12 & Asteraceae & Vernonia arborea Buch. & 8 & & & No \\
\hline \multirow[t]{5}{*}{13} & Euphorbiaceae & Macaranga recurvata Gage & 8 & & & No \\
\hline & Euphorbiaceae & Endospermum diadenum (Miq.) Airy Shaw & 8 & & & No \\
\hline & Euphorbiaceae & Macaranga pruinosa (Miq.) Müll.Arg & 8 & & & No \\
\hline & Euphorbiaceae & $\begin{array}{l}\text { Macaranga gigantea (Reichb.f. \& Zoll.) } \\
\text { Mull.Arg. }\end{array}$ & 17 & & & No \\
\hline & Moraceae & Artocarpus sp. & 8 & & & No \\
\hline \multirow[t]{3}{*}{14} & Fabaceae & Fordia brachybotrys Merr & & 1.200 & 5.000 & New \\
\hline & Fabaceae & Spatholobus ferrugineus (Zoll \& Moritzi) Benth. & & & 2.500 & New \\
\hline & Fagaceae & Lithocarpus lucidus (Roxb.) Rehder & 8 & 133 & & poor \\
\hline 15 & Celastraceae & Siphonodon celastrineus Griff & 8 & & & No \\
\hline 16 & Annonaceae & Hydnocarpus polypetala (Sloot.) Sleum. & 8 & & & No \\
\hline 17 & Chrysobalanaceae & Maranthes corymbosa $\mathrm{Bl}$ & 8 & & & No \\
\hline 18 & Lecythidaceae & Barringtonia macrostachya (Jack) Kurz. & 8 & & & No \\
\hline 19 & Malvaceae & Sterculia rubiginosa Zoll. & 8 & & & No \\
\hline 20 & Cannabaceae & Gironniera nervosa Planch. & 8 & 267 & & New \\
\hline 21 & Combretaceae & Terminalia foetidissima Griff & 8 & & 83 & poor \\
\hline 22 & Vitaceae & Leea indica (Burm.f.) Merr. & & 2.13 & 3.333 & New \\
\hline \multirow[t]{2}{*}{23} & Myrtaceae & $\begin{array}{l}\text { Syzygium palawanense (C.B.Rob.) Merr. \& } \\
\text { Perry. }\end{array}$ & & 667 & 1.667 & New \\
\hline & Myrtaceae & Rhodamnia cinerea Jack. & & 267 & & New \\
\hline 24 & Dilleniaceae & Dillenia reticulata King. & & 267 & & New \\
\hline 25 & Celastraceae & Siphonodon celastrineus Griff. & & 400 & & New \\
\hline 26 & Celastraceae & Bhesa paniculata Arn. & & 133 & & New \\
\hline 27 & Sterculiaceae & Leptonychia caudata (Wall. ex G.Don) Burret & & 267 & & New \\
\hline 28 & Myristicaceae & Myristica elliptica Wall. ex Hook.f. \& Thomson & & 267 & & New \\
\hline 29 & Sapotaceae & Palaquium dasyphyllum Pierre ex Dubard & & 267 & & New \\
\hline 30 & Olacaceae & Strombosia javanica Blume & & 245 & 1.667 & New \\
\hline 31 & Moraceae & Artocarpus sp. & & 133 & & No \\
\hline 32 & Salicaceae & Flacourtia rukam Zoll. \& Mor. & & 140 & & No \\
\hline 33 & Marantaceae & $\begin{array}{l}\text { Stachyphrynium repens (Körn.) Suksathan \& } \\
\text { Borchs. }\end{array}$ & & & 12.500 & New \\
\hline 34 & Passifloraceae & Adenia macrophylla (Blume) Koord & & & 5.000 & New \\
\hline 35 & Araceae & Epipremnum amplissimum (Schott) Engl. & & & 3.333 & New \\
\hline 36 & Lygodiaceae & Lygodium circinatum (Burm.) Sw. & & & 2.500 & New \\
\hline 37 & Poaceae & Cyrtococcum patens $(\mathrm{L})$. & & & 833 & New \\
\hline 38 & Dioscoreaceae & Dioscorea sp. & & & 833 & New \\
\hline 39 & Sapindaceae & Lepisanthes amoena (Hassk.) Leenh & & & 833 & New \\
\hline
\end{tabular}


Table 3. Regeneration status of trees in the conservation forest of the buffer zone of the Liquid Natural Gas Industry area, East Kalimantan

\begin{tabular}{|c|c|c|c|c|c|c|}
\hline \multirow[t]{2}{*}{ No } & \multirow[t]{2}{*}{ Family } & \multirow[t]{2}{*}{ Species } & \multicolumn{3}{|c|}{ Density (Ind/ha) } & \multirow{2}{*}{$\begin{array}{l}\text { Status } \\
\text { regeneration }\end{array}$} \\
\hline & & & Tree & Sapling & Seedling & \\
\hline \multirow[t]{6}{*}{1} & Dipterocarpaceae & Shorea johorensis Foxw & 42 & 92 & 7,692 & good \\
\hline & Dipterocarpaceae & Shorea smithiana Sym & 38 & - & - & No \\
\hline & Dipterocarpaceae & Shorea ovalis (Korth.) Bl & 17 & - & - & No \\
\hline & Dipterocarpaceae & Shorea grandiflora Brandis & 6 & - & - & No \\
\hline & Dipterocarpaceae & Shorea sp. & 2 & 92 & 192 & good \\
\hline & Dipterocarpaceae & Dryobalanops lanceolata Burck & 2 & - & - & No \\
\hline \multirow[t]{4}{*}{2} & Euphorbiaceae & Macaranga gigantea (Reichb.f. \& Zoll.) Mull.Arg & 44 & - & - & No \\
\hline & Euphorbiaceae & Croton griffithii Hook & 4 & 62 & 1,346 & good \\
\hline & Euphorbiaceae & Blumeodendron tokbrai (B1.) Kurz & 4 & - & - & No \\
\hline & Euphorbiaceae & Macaranga tanarius (L.) Mull.Arg & 2 & $215 ?$ & - & poor \\
\hline 3 & Lamiaceae & Vitex pinnata $\mathrm{L}$ & 40 & 62 & 2,308 & good \\
\hline \multirow[t]{6}{*}{4} & Moraceae & Artocarpus elasticus Reinw. ex Blume & 19 & 92 & - & poor \\
\hline & Moraceae & Ficus variegata Blume & 2 & $62 ?$ & 577 & good \\
\hline & Moraceae & Artocarpus lakoocha Roxb & 2 & $92 ?$ & - & poor \\
\hline & Moraceae & Artocarpus tamaran Becc & 2 & - & - & No \\
\hline & Moraceae & Ficus stricta (Miq.) Miq & 2 & - & - & No \\
\hline & Lauraceae & Phoebe sp. & 6 & - & - & No \\
\hline \multirow[t]{2}{*}{5} & Fagaceae & Lithocarpus sundaicus (Blume) Rehd. & 10 & - & $192 ? ?$ & No \\
\hline & Fagaceae & Lithocarpus blumeanus (Korth.) Rehd & 6 & - & - & No \\
\hline \multirow[t]{3}{*}{6} & Malvaceae & Sterculia rubiginosa Zoll. ex Miq & 12 & - & - & No \\
\hline & Malvaceae & Pterospermum javanicum Jungh & 8 & - & - & No \\
\hline & Malvaceae & Pentace triptera Mast & 2 & - & - & No \\
\hline \multirow[t]{2}{*}{7} & Lecythidaceae & Barringtonia macrostachya (Jack) Kurz & 12 & - & - & No \\
\hline & Lecythidaceae & Planchonia grandis Ridl & 15 & - & - & No \\
\hline \multirow[t]{5}{*}{8} & Fabaceae & Acacia auriculiformis A.Cunn. ex Benth & 13 & - & - & No \\
\hline & Fabaceae & Archidendron jiringa (Jack) Nielsen & 6 & 62 & 192 & good \\
\hline & Fabaceae & Dialium indum $\mathrm{L}$ & 6 & - & - & No \\
\hline & Fabaceae & Cassia siamea Lam & 4 & - & - & No \\
\hline & Fabaceae & Adenanthera borneensis Brace ex Prain & 2 & - & - & No \\
\hline \multirow[t]{5}{*}{9} & Myrtaceae & Syzygium sp. & 8 & 277 & 962 & good \\
\hline & Myrtaceae & Syzygium hirtum (Korth.) Merr. \& Perry & 4 & 62 & - & poor \\
\hline & Myrtaceae & Vernonia arborea Buch.-Ham. ex Buch.-Ham & 4 & - & - & No \\
\hline & Myrtaceae & Syzygium palawanense (C.B.Rob.) Merr. \& Perry & 2 & 31 & - & poor \\
\hline & Myrtaceae & Syzygium tenuicaudatum Merr. \& Perry & 2 & - & - & No \\
\hline \multirow[t]{2}{*}{10} & Burseraceae & Dacryodes rostrata (Bl.) H.J. Lam & 10 & 92 & - & poor \\
\hline & Burseraceae & Santiria grandiflora Kalkman & 6 & - & - & No \\
\hline 11 & Annonaceae & Polyalthia rumphii (B1.) Merr & 8 & - & - & No \\
\hline & Annonaceae & Polyalthia sp. & 4 & - & - & No \\
\hline 12 & Rubiaceae & Nauclea officinalis (Pierre ex Pitard) Merr. \& Chun & 4 & 31 & 192 & good \\
\hline & Rubiaceae & Gardenia tubifera Wall & 2 & - & - & No \\
\hline 13 & Cannabaceae & Gironniera subaequalis Planch & 4 & - & 3,462 & No \\
\hline 14 & Sapotaceae & Palaquium dasyphyllum Pierre ex Dubard, Bull & 6 & 92 & - & poor \\
\hline 15 & Ebenaceae & Diospyros borneensis Hiern & 6 & 92 & - & poor \\
\hline 16 & Anacardiaceae & Campnosperma coriaceum (Jack) Hall.f & 4 & - & - & No \\
\hline & Anacardiaceae & Buchanania arborescens (Blume) Blume & 4 & - & - & No \\
\hline & Anacardiaceae & Gluta sp & 2 & - & - & No \\
\hline & Anacardiaceae & Dracontomelon costatum $\mathrm{Bl}$ & 2 & - & - & No \\
\hline & Anacardiaceae & Semecarpus $\mathrm{sp}$. & 2 & - & - & No \\
\hline 17 & Oxalidaceae & Sarcotheca macrophylla Blume & 4 & 92 & - & poor \\
\hline 18 & Irvingiaceae & Irvingia malayana Oliv & 2 & - & - & No \\
\hline 19 & Hypericaceae & Cratoxylum formosum (Jack) Benth. \& Hook & 4 & - & - & No \\
\hline 20 & Thymelaeaceae & Aquilaria malaccensis Lamk & 2 & - & - & No \\
\hline 21 & Apocynaceae & Cerbera manghas $\mathrm{L}$ & 4 & - & - & No \\
\hline 22 & Celastraceae & Lophopetalum beccarianum Pierre \& Ridl & 2 & - & - & No \\
\hline 23 & Meliaceae & Swietenia macrophylla King & 4 & - & - & No \\
\hline & Meliaceae & Aglaia crassinervia Kurz ex Hiern & 2 & - & 192 & No \\
\hline & Meliaceae & Walsura pachycaulon T.P.Clarck & 2 & - & - & No \\
\hline & Meliaceae & Carapa guianensis Aubl & 2 & - & - & No \\
\hline 24 & Theaceae & Schima wallichii (DC) Korth & 2 & - & - & No \\
\hline 25 & Hypericaceae & Cratoxylum sumatranum (Jack) Blume & 4 & - & - & No \\
\hline 26 & Myristicaceae & Myristica smythiesii J.Sinclair, Gard & 2 & - & - & No \\
\hline & Myristicaceae & Knema hirtella W.J.deWilde & 2 & 62 & - & poor \\
\hline
\end{tabular}




\begin{tabular}{|c|c|c|c|c|c|c|}
\hline 27 & Dilleniaceae & Dillenia borneensis Hoogl & 2 & - & 385 & \\
\hline \multirow[t]{4}{*}{28} & Annonaceae & $\begin{array}{l}\text { Cyathocalyx deltoideus (Airy Shaw) R.J. Wang \& } \\
\text { R.M.K. Saunders }\end{array}$ & 2 & 62 & - & Poor \\
\hline & Annonaceae & Miliusa macropoda $\mathrm{Miq}$ & 2 & - & - & No \\
\hline & Annonaceae & $\begin{array}{l}\text { Cyathocalyx magnifructus } \\
\text { R.M.K.Saunders }\end{array}$ & 2 & - & - & No \\
\hline & Annonaceae & Cyathocalyx virgatus (B1.) King & 2 & - & - & No \\
\hline \multirow[t]{2}{*}{29} & Rubiaceae & Neonauclea calycina (DC) Merr & 2 & - & - & No \\
\hline & Rubiaceae & Diplospora malaccensis Hook & 2 & 31 & & Poor \\
\hline \multirow[t]{4}{*}{30} & Lauraceae & Cinnamomum parthenoxylon (Jack) Meisn & 2 & - & - & No \\
\hline & Lauraceae & Cinnamomum iners Reinw. ex Blume & 2 & - & - & No \\
\hline & Lauraceae & Litsea $\mathrm{sp}$ & 2 & 31 & - & Poor \\
\hline & Lauraceae & Cryptocarya impressa Miq & 2 & - & - & No \\
\hline 31 & Rosaceae & Prunus beccarii (Ridl.) Kalkman & 2 & - & - & No \\
\hline 32 & Phyllanthaceae & Baccaurea tetrandra (Baill.) Mull.Arg & 2 & - & - & No \\
\hline 33 & Chrysobalanaceae & Licania splendens (Korth.) Prance & 2 & - & - & No \\
\hline
\end{tabular}

The average Margallef $(\mathrm{R})$ richness index values for the level of trees, saplings and seedlings in conservation forests in the buffer zone ranged between 3.48 and 5.77 (medium to high). In Bontang urban forest, the species value (R) for the tree level was 7.25 (high), for sapling level was 4.66 (medium) and for seedling level was 3.16 (low). This condition illustrates that in urban forests there were more species richness of trees than seedlings and saplings. The Bontang urban forest in the company area had more tree species than conservation forests in the Industrial buffer zone.

The tree-level biodiversity ( $\left.H^{\prime}\right)$ index value in each sample plot was classified as moderate to high with a value of 1.5. > 3, seedlings and saplings were classified as moderate with H' values between 2 to 3 . Dominance index values $(C)$ for all growth stages were low with values of 0 $<\mathrm{C}<0.5$. Evenness index value (e) of all growth stages were found to be almost even with e values between 0.76 and 0.96 . This may be due to the relatively similar number of specify species of forest fragments, so that competition between species was relatively low.

\section{Regeneration status}

According to the regeneration status based categories proposed by Malik and Bhatt (2016), among 76 species of trees recorded from the conservation forest of the buffer zone area, 8 species $(10.52 \%)$ showed good regeneration status, 13 species $(17.10 \%)$ showed poor status and 55 species $(72.36 \%)$ showed no regeneration status. Tree species that showed good regeneration included Shorea johorensis, Shorea sp., Croton griffithii, Vitex pinnata, Ficus variegata, Archidendron jiringa, Syzygium sp. and Nauclea officinalis. Among the seven Dipterocarpaceae species, only two species, namely Shorea sp. and Shorea johorensis, had good regeneration status.

Among the 53 tree species found in Bontang urban forest, none showed good regeneration status, 28 species $(52.83 \%)$ showed no regeneration status, 3 species $(5,669 \%)$ showed poor regeneration and 22 species $(41.50 \%)$ belonged to generation of new species. All the 7 Dipterocarpaceae species recorded including Vatica umbonata, Shorea leprosula, S.ovalis, S.johorensis, S.smithiana, S.elliptica and Anisoptera costata showed no regeneration status (Table 2, Table 3).
The problem of lack of regeneration, especially for Dipterocarpaceae plants, is in line with Yasman (1994) and Smits (1986) stating that Dipterocarpaceae seeds are recalcitrant so they cannot be stored for a long time, while erratic flowering and fertilization ranges from 4-5 years once or every 5-13 years. Due to this condition, seeds are not available every year. Therefore, it was difficult to find seedlings of Dipterocarpaceae species. Inability to find any mother trees that was flowering or bearing fruit is an indicator that the trees are facing difficulties in flowering in urban forests, and also in conservation forest areas of the buffer zone. Among the seven species of Dipterocarpaceae, only two seedling species were found, namely Shorea sp. and Shorea johorensis.

\section{Discussion}

Understanding the ecology of the old forest and maintaining its distinctive biological diversity requires a very long and extensive study of its various aspects. The process of natural regeneration of forest ecosystems is a parameter that plays an important role in the sustainability of the structure and composition of its plant species. The long history of the formation of forest fragments in Bontang City has included the environment of the liquefied natural gas refinery industry since the 1970s. Without being disturbed by the company, this forest exists as representative of lowland tropical forest in East Kalimantan. Tree density is 562 Ind./ha, sapling density is 7,933 Ind./ha and seedlings and understorey density is 80,625 Ind./ha. The average species richness of trees in the urban forest is high $(\mathrm{R}>5)$, but the sapling is in a moderate status $(\mathrm{R}=4.66)$ and the seedling level is low $(\mathrm{R}<3.16)$. The average tree diversity index value is in high category $\left(\mathrm{H}^{\prime}>3\right)$ and the sapling and seedling diversity conditions are in moderate status ( $\mathrm{H}$ ' between $2-3$ ). The average value of evenness is quite even (e between $0.76-0.96$ ), but the dominance index is low for all tree stratification, sapling and seedlings $(\mathrm{C}$ values $<\mathrm{C}<0.5)$.

Some tree species show lack of regeneration in the Bontang urban forest area which is in the liquefied natural gas refinery industry, namely Planchonia grandis Ridl, Alstonia scholaris (L.) R.Br, Cratoxylum formosum (Jack) Benth. \& Hook.f. ex Dyer., Vatica umbonata (Hook. f.) Burck, Shorea smithiana Sym, Shorea leprosula Miq, 
Anisoptera costata North. Shorea ovalis Blume, Schima wallichii (DC.) Korth, Licania splendens (Korth.) Prance \& Kosterm, Breonia chinensis (Lam.) Capuron, Tarenna costata Merr, Actinodaphne macrophylla (Blume) Nees.Bhesa paniculata Arn.Dacryodes rostrata (B1.) HJ Lam,Garcinia beccarii Pierre. Vernonia arborea Buch. Macaranga recurvata Gage, Endospermum diadenum (Miq.) Airy Shaw, Macaranga pruinosa (Miq.) Müll. Arg, Macaranga gigantea (Reichb.f. \& Zoll.) Mull. Arg., Artocarpus sp. Siphonodon celastrineus Griff, Polypetala hydnocarpus (Sloot.) Sleum., Maranthes corymbosa Bl, Barringtonia macrostachya (Jack) Kurz., Sterculia rubiginosa Zoll.

The aforementioned conditions illustrate that the regeneration process of native tree species is hampered by various factors including the availability of seeds, seed predation, seed dispersal, and seed formation (Shono et al. 2006). Floristic and structural composition of forests will change from one community state to another community state simultaneously with the ability to compete with the existing seedlings to become their next-generation (Barker and Kirkpatrick 1994). Natural regeneration can take place through the formation of seeds and shoots from stumps to produce high-quality forests with high biodiversity (Yang et al. 2014). Factors that effectively determine the success of regeneration include seed availability, soil cover, seed growth status, tree stand cover, tree canopy average, organic matter content in the soil, soil reaction, rainfall and variation in growth requirements (Özel et al. 2010; Smith et al. 2016). Meanwhile, Khaine et al. (2018) reported that in Myanmar's tropical rainforest the average annual rainfall (abiotic) factors, as well as ecosystem complexity, density, species richness, and overstory were found to be the most influential factors for density and diversity of natural forest regeneration.

From the description above, to make older forests to regenerate, there must be enough seeds that can grow into seedlings. This condition requires seed spreading agents that can move from one forest fragment to other forest fragments. Besides, the availability of seedlings is often incidental and depends on the condition of the soil, remaining standing density, the composition of stand species that produce their seeds, and climatic conditions when seeds are available and growing, light intensity, and shade tolerance. The condition of isolated urban forests from their parent habitat makes these agents may not be available in sufficient quantities. It is considered to cause the unavailability of seedlings and saplings of the same species as the parent tree.

In general, the study has shown that Bontang urban forest located within the area of the liquefied natural gas refinery company is in very poor or low natural regeneration status. The low level of natural regeneration of certain species, theoretically shows that the population of these species is in a phase of degradation and can threaten the sustainability of species in the future. The density of seedlings and saplings of each species varies, resulting in different regeneration patterns. Based on these data, it can be concluded that natural regeneration alone may not be sufficient to maintain the desired stock of each species to be maintained, and immediate restoration steps must be taken to assist the natural regeneration process.

\section{ACKNOWLEDGEMENTS}

The authors would like to thank the Dean of Faculty of Mathematics and Sciences, Mulawarman University for funding this research, as well as all those who have helped both in the field and in the laboratory.

\section{REFERENCES}

Barker PCJ, Kirkpatrick JB. 1994. Phyllocladus aspleniifolius: variability in the population structure, the regeneration niche and dispersion patterns in Tasmanian forests. Aust J Bot 42(2): 163-190.

Cox GW. 1976. Laboratory Manual of General Biology. San Diego State University \& Win. C. Brown Company Publisher, Dubuque, IO.

Dhar U, Rawal RS, Samant SS. 1997. Structural diversity and representativeness of forest vegetation in a protected area of Kumaun Himalaya, India: Implications for conservation. Biodivers Conserv 6(8): 1045-1062.

Khaine I, Woo SY, Kwak MJ, Lee SH, Je SM, You H, Lee T, Jang J, Lee HK, Cheng HC, Park JH, Lee E, Li Y, Kim H, Lee JK, Kim J. 2018. Factors affecting natural regeneration of tropical forests across a precipitation gradient in Myanmar. Forests 9(143). DOI:10.3390/f9030143 www.mdpi.com/journal/forests.

Kusmana C. 1997. Metode Survey Vegetasi. Institut Pertanian Bogor Press, Bogor. [Indonesian]

Kusmana C, Susati S. 2015. Komposisi dan struktur tegakan hutan alam di hutan pendidikan Gunung Walat, Sukabumi. J Silv Trop (5)3: 210207. [Indonesian]

Malik ZA, Bhatt AB. 2016. Regeneration status of tree species and survival of their seedlings in Kedarnath Wildlife Sanctuary and its adjoining areas in Western Himalaya, India. Trop Ecol 57(4): 677-690.

Mishra AK, Behera SK, Singh K. 2013. Influence of abiotic factors on community structure of understory vegetation in moist deciduous forests of north India. For Sci Pract 15: 261-273.

Mueller-Dombois D, Ellenberg H. 1974. Aims and Methods of Vegetation Ecology. John Wiley and Sons, New York

Ngo KM, Davies S, Hassan NFN, Lum S. 2016. Resilience of a forest fragment exposed to long-term isolation in Singapore. Plant Ecol Divers 9(4): 397-407. DOI: 10.1080/ 17550874 .2016.1262924.

Özel HB, Ertekin M, Yilmaz M, Kirdar E. 2010. Factors affecting the success of natural regeneration in Oriental Beech (Fagus orientalis Lipsky) forests in Turkey. Acta Silv Lign Hung 6: 149-160.

Pudyatmoko S, Djoko M, Suwarna H. 1997. Pengelompokan komunitas hutan di Taman Nasional Baluran Jawa Timur. Bull Kehut No.31/1997. [Indonesian]

Shono K, Davies SJ, Chua YK.2006. Regeneration of native plant species in restored forests on degraded lands in Singapore. For Ecol Manag 237: 574-582.

Sing SP, Tewart JC, Yadav S, Ralhan PK.1986. Population structure of tree species in forests as an indicator of regeneration and future stability. Proc Indian Acad Sci (Plant Sci) 6(69): 443-455. India, December.

Smits WTM. 1986. Pedoman Sistem Cabutan Bibit Dipterocarpaceae. Balai Penelitian Kehutanan Samarinda. [Indonesian]

Smith AL, Blanchard W, Blair DP, McBurney L, Banks SC, Driscoll DA, Lindenmayer DB. 2016. The dynamic regeneration niche of a forest following a rare disturbance event. Divers Distrib 22: 457-467.

Yasman I. 1994. Dasar-Dasar Pengenalan Anakan Dipterocarpaceae. Balai Penelitian Kehutanan Samarinda. (Edisi khusus). No. 10: 1-23. [Indonesian]

Yang X, Yan D, Liu C. 2014. Natural regeneration of trees in three types of afforested stands in the Taihang Mountains, China. PLoS ONE 9(9): e108744. DOI:10.1371/journal.pone.0108744.

Zuhud EAM, Agus H, Dones R,Siswoyo, Purwowidodo, Yeni AM, Cahyo W.1995. Kegiatan Penataan Desain Botanical Garden PT Badak Co, Bontang Kalimantan Timur. Kerjasama antara PT Badak Co dengan Fakultas Kehutanan IPB, Bogor. [Indonesian] 